\title{
FIXED POINTS BY A NEW ITERATION METHOD
}

\author{
SHIRO ISHIKAWA
}

\begin{abstract}
The following result is shown. If $T$ is a lipschitzian pseudo-contractive map of a compact convex subset $E$ of a Hilbert space into itself and $x_{1}$ is any point in $E$, then a certain mean value sequence defined by $x_{n+1}=\alpha_{n} T\left[\beta_{n} T x_{n}+\left(1-\beta_{n}\right) x_{n}\right]+\left(1-\alpha_{n}\right) x_{n}$ converges strongly to a fixed point of $T$, where $\left\{\alpha_{n}\right\}$ and $\left\{\beta_{n}\right\}$ are sequences of positive numbers that satisfy some conditions.
\end{abstract}

It was recently shown in [1] that a mean value iteration method is available to find a fixed point of a strictly pseudo-contractive map. In this paper we shall prove that a certain sequence of points which is iteratively defined converges always to a fixed point of a lipschitzian pseudo-contractive map. For the definitions of a strictly pseudo-contractive map and a pseudo-contractive map in a Hilbert space, see, for example, [3].

THEOREM. If $E$ is a convex compact subset of a Hilbert space $H, T$ is a lipschitzian pseudo-contractive map from $E$ into itself and $x_{1}$ is any point in $E$, then the sequence $\left\{x_{n}\right\}_{n=1}^{\infty}$ converges strongly to a fixed point of $T$, where $x_{n}$ is defined iteratively for each positive integer $n$ by

$$
x_{n+1}=\alpha_{n} T\left[\beta_{n} T x_{n}+\left(1-\beta_{n}\right) x_{n}\right]+\left(1-\alpha_{n}\right) x_{n},
$$

where $\left\{\alpha_{n}\right\}_{n=1}^{\infty}$ and $\left\{\beta_{n}\right\}_{n=1}^{\infty}$ are sequences of positive numbers that satisfy the following three conditions:

$$
\begin{aligned}
0 \leqq \alpha_{n} \leqq \beta_{n} \leqq & 1 \text { for all positive integers } n, \\
& \lim _{n \rightarrow \infty} \beta_{n}=0, \\
& \sum_{n=1}^{\infty} \alpha_{n} \beta_{n}=\infty .
\end{aligned}
$$

As a particular case, we may choose for instance $\alpha_{n}=\beta_{n}=n^{-1 / 2}$.

Received by the editors November 24, 1972 and, in revised form, March 28, 1973 and August 16, 1973.

AMS (MOS) subject classifications (1970). Primary 47H10; Secondary 40A05.

Key words and phrases. Iteration method, pseudo-contractive map.

(c) American Mathematical Society 1974 
Proof. We have, for any $x, y, z$ in a Hilbert space $H$ and a real number $\lambda$,

$$
\begin{aligned}
\| \lambda x+ & (1-\lambda) y-z\left\|^{2}=\right\| \lambda(x-y)+y-z \|^{2} \\
= & \lambda^{2}\|x-y\|^{2}+\|y-z\|^{2}+2 \lambda \operatorname{Re}(x-y, y-z) \\
= & \lambda^{2}\|x-y\|^{2}+\|y-z\|^{2} \\
& \quad+\lambda \operatorname{Re}\left[\left(\|x\|^{2}-2(x, z)+\|z\|^{2}\right)\right. \\
& \left.\quad-\left(\|x\|^{2}-2(x, y)+\|y\|^{2}\right)-\left(\|z\|^{2}-2(z, y)+\|y\|^{2}\right)\right] \\
& =\lambda^{2}\|x-y\|^{2}+\|y-z\|^{2}+\lambda\left(\|x-z\|^{2}-\|x-y\|^{2}-\|y-z\|^{2}\right) \\
= & \lambda\|x-z\|^{2}+(1-\lambda)\|y-z\|^{2}-\lambda(1-\lambda)\|x-y\|^{2} .
\end{aligned}
$$

Since $T$ is pseudo-contractive, for any $x, y$ in $E$,

$$
\|T x-T y\|^{2} \leqq\|x-y\|^{2}+\|(I-T) x-(I-T) y\|^{2},
$$

where $I$ is an identity map.

From the assumption that $T$ is lipschitzian, we also have that there is a positive number $L$ such that

$$
\|T x-T y\| \leqq L\|x-y\| \text { for any } x, y \text { in } E .
$$

From Schauder's fixed point theorem, $F(T)$, the set of fixed points of $T$, is nonempty since $E$ is a convex compact set and $T$ is continuous. Let $p$ denote any point of $F(T)$.

We have, from (5) in which $\lambda$ stands for $\alpha_{n}$ or $\beta_{n}$, the following three equalities, namely

and

$\left\|x_{n+1}-p\right\|^{2}=\left\|\alpha_{n} T\left[\beta_{n} T x_{n}+\left(1-\beta_{n}\right) x_{n}\right]+\left(1-\alpha_{n}\right) x_{n}-p\right\|^{2}$

8) $\quad=\alpha_{n}\left\|T\left[\beta_{n} T x_{n}+\left(1-\beta_{n}\right) x_{n}\right]-p\right\|^{2}+\left(1-\alpha_{n}\right)\left\|x_{n}-p\right\|^{2}$

$$
-\alpha_{n}\left(1-\alpha_{n}\right)\left\|T\left[\beta_{n} T x_{n}+\left(1-\beta_{n}\right) x_{n}\right]-x_{n}\right\|^{2},
$$

$$
\begin{aligned}
\left\|\beta_{n} T x_{n}+\left(1-\beta_{n}\right) x_{n}-p\right\|^{2}= & \beta_{n}\left\|T x_{n}-p\right\|^{2}+\left(1-\beta_{n}\right)\left\|x_{n}-p\right\|^{2} \\
& -\beta_{n}\left(1-\beta_{n}\right)\left\|T x_{n}-x_{n}\right\|^{2}
\end{aligned}
$$

$$
\begin{aligned}
\| \beta_{n} T x_{n}+ & \left(1-\beta_{n}\right) x_{n}-T\left[\beta_{n} T x_{n}+\left(1-\beta_{n}\right) x_{n}\right] \|^{2} \\
= & \beta_{n}\left\|T x_{n}-T\left[\beta_{n} T x_{n}+\left(1-\beta_{n}\right) x_{n}\right]\right\|^{2} \\
& +\left(1-\beta_{n}\right)\left\|x_{n}-T\left[\beta_{n} T x_{n}+\left(1-\beta_{n}\right) x_{n}\right]\right\|^{2} \\
& -\beta_{n}\left(1-\beta_{n}\right)\left\|T x_{n}-x_{n}\right\|^{2} .
\end{aligned}
$$

Moreover from (6) we have the following two inequalities, namely

$$
\begin{aligned}
& \left\|T\left[\beta_{n} T x_{n}+\left(1-\beta_{n}\right) x_{n}\right]-p\right\|^{2}=\left\|T\left[\beta_{n} T x_{n}+\left(1-\beta_{n}\right) x_{n}\right]-T p\right\|^{2} \\
& \leqq \\
& \quad\left\|\beta_{n} T x_{n}+\left(1-\beta_{n}\right) x_{n}-p\right\|^{2} \\
& \quad+\left\|\beta_{n} T x_{n}+\left(1-\beta_{n}\right) x_{n}-T\left[\beta_{n} T x_{n}+\left(1-\beta_{n}\right) x_{n}\right]\right\|^{2},
\end{aligned}
$$


and

$$
\left\|T x_{n}-p\right\|^{2}=\left\|T x_{n}-T p\right\|^{2} \leqq\left\|x_{n}-p\right\|^{2}+\left\|x_{n}-T x_{n}\right\|^{2} .
$$

Performing the calculation according to (8) $+\alpha_{n}[(9)+(10)+(11)+$ $\left.\beta_{n} \cdot(12)\right]$ side by side and eliminating common terms on both sides of the resulting inequality, we have

$$
\begin{aligned}
\left\|x_{n+1}-p\right\|^{2} \leqq & \left\|x_{n}-p\right\|^{2}-\alpha_{n} \beta_{n}\left(1-2 \beta_{n}\right)\left\|T x_{n}-x_{n}\right\|^{2} \\
& +\alpha_{n} \beta_{n}\left\|T x_{n}-T\left[\beta_{n} T x_{n}+\left(1-\beta_{n}\right) x_{n}\right]\right\|^{2} \\
& -\alpha_{n}\left(\beta_{n}-\alpha_{n}\right)\left\|x_{n}-T\left[\beta_{n} T x_{n}+\left(1-\beta_{n}\right) x_{n}\right]\right\|^{2},
\end{aligned}
$$

and it follows from condition (2) that

$$
\begin{aligned}
\left\|x_{n+1}-p\right\|^{2} \leqq & \left\|x_{n}-p\right\|^{2}-\alpha_{n} \beta_{n}\left(1-2 \beta_{n}\right)\left\|T x_{n}-x_{n}\right\|^{2} \\
& +\alpha_{n} \beta_{n}\left\|T x_{n}-T\left[\beta_{n} T x_{n}+\left(1-\beta_{n}\right) x_{n}\right]\right\|^{2} .
\end{aligned}
$$

Now since $T$ is lipschitzian, we have, from (7),

$$
\left\|T x_{n}-T\left[\beta_{n} T x_{n}+\left(1-\beta_{n}\right) x_{n}\right]\right\| \leqq L \beta_{n}\left\|T x_{n}-x_{n}\right\| .
$$

We have finally from (13) and (14),

$$
\left\|x_{n+1}-p\right\|^{2} \leqq\left\|x_{n}-p\right\|^{2}-\alpha_{n} \beta_{n}\left(1-2 \beta_{n}-L^{2} \beta_{n}^{2}\right)\left\|T x_{n}-x_{n}\right\|^{2} .
$$

Therefore adding these inequalities with $m, m+1, \cdots, n$ for $n$, we derive the following inequality

$$
\left\|x_{n+1}-p\right\|^{2} \leqq\left\|x_{m}-p\right\|^{2}-\sum_{k=m}^{n} \alpha_{k} \beta_{k}\left(1-2 \beta_{k}-L^{2} \beta_{k}^{2}\right)\left\|T x_{k}-x_{k}\right\|^{2},
$$

from which we have

$$
\sum_{k=m}^{n} \alpha_{k} \beta_{k}\left(1-2 \beta_{k}-L^{2} \beta_{k}^{2}\right)\left\|T x_{k}-x_{k}\right\|^{2} \leqq\left\|x_{m}-p\right\|^{2}-\left\|x_{n+1}-p\right\|^{2} .
$$

From condition (3), there is some positive integer $N$ such that $2 \beta_{k}+$ $L^{2} \beta_{k}^{2}<\frac{1}{2}$ for all integers $k \geqq N$. Then if $m$ is larger than $N$, we can get the following inequality

$$
\frac{1}{2} \sum_{k=m}^{n} \alpha_{k} \beta_{k}\left\|T x_{k}-x_{k}\right\|^{2} \leqq\left\|T x_{m}-p\right\|^{2}-\left\|T x_{n+1}-p\right\|^{2} .
$$

The last member is bounded since $E$ is a bounded set. Therefore the series on the left hand side is bounded. From condition (4) this should imply that $\lim _{\inf _{n \rightarrow \infty}}\left\|T x_{n}-x_{n}\right\|=0$, which in turn implies from the compactness of $E$ that there is a subsequence $\left\{x_{n_{i}}\right\}_{i=1}^{\infty}$ that converges to a certain point $q$ of $F(T)$.

Since $q$ is a fixed point of $T$, from (15) we see that if $n \geqq N$,

$$
\left\|x_{n+1}-q\right\| \leqq\left\|x_{n}-q\right\| .
$$


Let $\varepsilon$ be any positive number. Then there is an $n_{i 0}$ such that $\left\|x_{n_{i 0}}-q\right\| \leqq \varepsilon$ and $n_{i 0} \geqq N$. Hence from (16), $\left\|x_{n}-q\right\| \leqq \varepsilon$ for $n \geqq n_{i 0}$.

This completes the proof of the theorem.

The author wishes to express his sincere thanks to Professor H. Fujita and Professor T. Kawata for their kind suggestions.

\section{REFERENCES}

1. G. G. Johnson, Fixed points by mean value iterations, Proc. Amer. Math. Soc. 34 (1972), 193-194. MR 45 \#1006.

2. W. R. Mann, Mean value methods in iteration, Proc. Amer. Math. Soc. 4 (1953), 506-510. MR 14, 988.

3. F. E. Browder and W. V. Petryshyn, Construction of fixed points of nonlinear mappings in Hilbert spaces, J. Math. Anal. Appl. 20 (1967), 197-228. MR 36 \#747.

4. R. L. Franks and R. P. Marzec, $A$ theorem on mean value iterations, Proc. Amer. Math. Soc. 30 (1971), 324-326. MR 43 \#6375.

Faculty of Engineering, Department of Electrical Engineering, Keio UniVERSITY, YOKOHAMA, JAPAN 